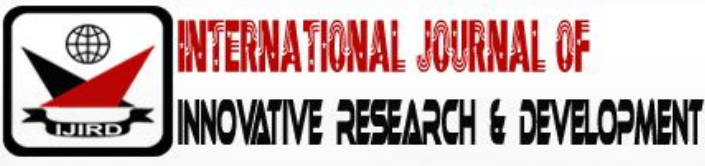

ISSN 2278 - 0211 (Online)

\section{Comparative Analysis of the Re-Convergence Ability of RIP, OSPF and EIGRP Routing Protocols}

\author{
Richard Kofi Annan \\ Assistant Lecturer, Department of UMaT, University of Mines and Technology, Ghana \\ Richard Ofosu Amoako \\ Student, Department of Computer Science and Engineering, University of Mines and Technology, Ghana \\ Jonas Tawiah Agyepong \\ Research and Teaching Assistant, Department of UMaT, University of Mines and Technology, Ghana
}

\begin{abstract}
:
Computer networks is one of the widely used communication medium for systems remote interactions; and one of the rapidly growing technology since its abstract was first proposed. Routing has become the means for selecting a path for traffic in a network, or between multiple networks. The two types of routing protocols in existence include: static and dynamic routing; however this study pivots on the dynamic ones which include EIGRP, OSPF and RIP. These routing protocols running on routers continuously exchange network status between each other in order to move packets from one network-domain to the other. After the routing information distribution, the state of convergence is achieved. Any alteration within the network topology affects the distributed routing information; which breaks the convergence. Hence, the need for re-convergence to match the updated network topology. During the time elapsed for re-convergence, packets traversing from one network domain to the other are loss. This study analyzes and performs a comparative analysis test for the different routing protocols namely: EIGRP, OSPF and RIP with respect to timely re-convergence ability with minimal packet loss using a designed network prototype. The analysis revealed that in the event of a convergence break, OSPF has the timely re-convergence ability with minimal packet loss rate followed by EIGRP and RIP.
\end{abstract}

Keywords: RIP, OSPF, EIGRP, routing, re-convergence, routing protocol

\section{Introduction}

Computer networks is one of the widely used communication medium for remote interactions; and one of the rapidly growing technology since its abstract was first proposed. Conventionally, the Internet Protocol (IP) network was first developed for military network communication and higher education research (Tewari \& Kumar, 2014). According to Tewari and Kumar (2014), IP networks could be envisaged as large graph with network nodes as vertices and transmission lines as edges. On such a graph, data in the form packets traverse over the edges from the vertices representing multiple network domains. This process has been described as routing by Tewari and Kumar (2014). According to Rathi and Singh (2015), routers and layer 3 switches are mainly used for this purpose.

Routing could be done statically or dynamically. Static routing involves the developing, maintaining and updating routing table manually such that a change in network topology affects its routing information (Gouveira, Pioro, \& Rak, 2016). On the other hand, dynamic routing uses routing protocols such that routing information is not highly dependent on the network topology being static (Rathi \& Singh, 2015). Routing in networks typically engages a rather involving collection of algorithms from which the protocols emanate. Some well-known routing algorithms used to develop routing protocols could be exemplified as the Distance Vector Routing (DVR) and Link State Routing (LSR) algorithms. Studies of Rathi and Singh, (2015) show that in DVR algorithm, a network domain node (router) knows the length of the shortest-path distance (edge) from each of its immediate neighbor; and to every destination node in the network. The network domain nodes use the shortest path distance information from leant nodes to compute its own distance and next reachable node to each destination. Routing Information Protocols (RIP) and Enhanced Interior Gateway Routing Protocol (IEGRP) are good illustrations of the routing protocols emanating from the DVR algorithm. Contrary to the DVR algorithm, the LSR algorithm maintains a complete view of the network topology with the metrics for each edge (link) (Cho \& Lee, 2011). Hussain and Jamwal (2016) define the metrics in terms of cost of communication, length of the path, reliability of the link and the likes. A node broadcast regularly the link metrics information to all other nodes. Once a link obtains the information, it updates its view of the topology and 
apply the shortest path algorithm to choose the next reachable network domain node for each destination. Open Shortest Path First (OSPF) and Intermediate System to Intermediate System (IS-IS) exemplifies the routing protocols derived from LSR algorithms.

The routing protocols (i.e. OSPF, RIP, EIGRP, IS-IS) work more or less independently; and yet support each other by exchanging routing information. This exchange of information occurs on the entire topology, making each network domain node gain knowledge of routes from others to form convergence amongst domains. By so doing a change in the topology does not affect the routing process as typical of dynamic routing compared to statically configuring the routes. An alteration within the network breaks the domain convergence; however, the configuration of routing protocols on domain nodes allows them to update their routing to table to match the new topology. Hence re-converging the updated network topology known as reconvergence. During the time elapsed for re-convergence, packets traversing the edges (link) from one network domain to the other are loss.

In literature, several studies have done performance evaluation on dynamic routing protocols with respect to routing updates, number of next hop updates, link delays and utilization and convergence. However, far little attention has been paid to re-convergence ability of the dynamic routing protocols. This study attempts to analyze and perform a comparative analysis for the dynamic routing protocols RIP, OSPF and EIGRP with respect to their re-convergence ability limiting packet loss and short elapsed time.

\section{Literature Review}

The Routing algorithms give rise to design routing protocols which are used to investigate the optimal path for data communication between network nodes. Tewari and Kumar (2014), points out that routing protocols are used to direct packets via network layer from a source node to valid destination as typically known as routing. Lee et al., (2001), draw attention to two fundamental routing algorithms; thus, Distance Vector Routing Algorithm (DVRA) and Link State Routing Algorithm (LSRA) which are used to design some OSI layer 3 routing protocols including the likes of RIP, OSPF and EIGRP.

\subsection{Routing Information Protocol (RIP)}

Verma and Bhardwaj (2016) mentions that, routing Information Protocol (RIP) is one of the most generic routing protocols for smaller networks which of the same or similar kind. As a distance-vector routing protocol, RIP is used by routers to share topology information from time to time by sending out routing table details to neighbouring routers every 30 seconds. These neighbouring routers in turn extend the information to other routers until they reach network convergence. RIP uses the hop count metric with the maximum limit of 15 hops; thereby, anything beyond that is inaccessible. As a result of the hops limitations this, RIP is not suitable for large and complex networks. A typical advantage of RIP would be its simplicity compared to other protocols; whereas its setback includes; the hop limitation, slow coverage and using only hop counts metrics to calculate best route.

\subsection{Enhanced Interior Gateway Routing Protocol (EIGRP)}

According to Rakheja et al., (2012), Enhanced Interior Gateway Routing Protocol (EIGRP) is also a based on DVRA which exchanges routing table information with neighbouring routers in an autonomous system. Unlike RIP, EIGRP shares routing table information that is not available in the neighbouring routers, thereby limiting or decreasing undesirable traffic transmitted through routers. EIGRP is an enhanced version of IGRP and uses Diffusing Update Algorithm (DUAL), which reduces the time taken for network intersection and improves operational efficiency. EIGRP was a proprietary protocol from Cisco ${ }^{\circledR}$, which was later made an Open Standard in 2013 (Lee et al., 2001). There are four basic components to operate EIGRP, which include: neighbour discovery or recovery, reliable transport protocol, DUAL finite state machine and protocol dependent module (Bansal \& Goel, 2017). The support of authentication and fast convergence attributes the merits of using EIGRP; however, its sole ownership of CISO would be demerit.

\subsection{Open Shortest Path First (OSPF)}

Open Shortest Path First (OSPF), is an LSRA based routing protocol often used in large networks for their Autonomous System (AS) networks. According to Quoitin and Uhlig (2005), an autonomous system is a collection of network prefixes within the internet. A collection of network prefixes is grouped into an autonomous system (AS). An AS is an individual network on the internet. Each network, or AS, has an autonomous system number (ASN). OSPF assembles link state information from available nodes and determines the routing table information to forward packets based on the destination IP address (Rakheja et al., 2012). This happens by creating a topology map for the network. Any change in the link is immediately identified and the information is forwarded to all other nodes; thereby, implying they also having the same routing table information in all nodes. Wang, Lui and Wang (2011), claims OSPF is used in complex networks that are modular for enhanced network administration and traffic optimization. Some of the advantages of OSPF would be the no limitation of hop counts and loop free route are always determined; however, it requires much more memory and its configuration is involving (Wang, Lui, $\&$ Wang, 2011). 


\section{Resources and Methods Used}

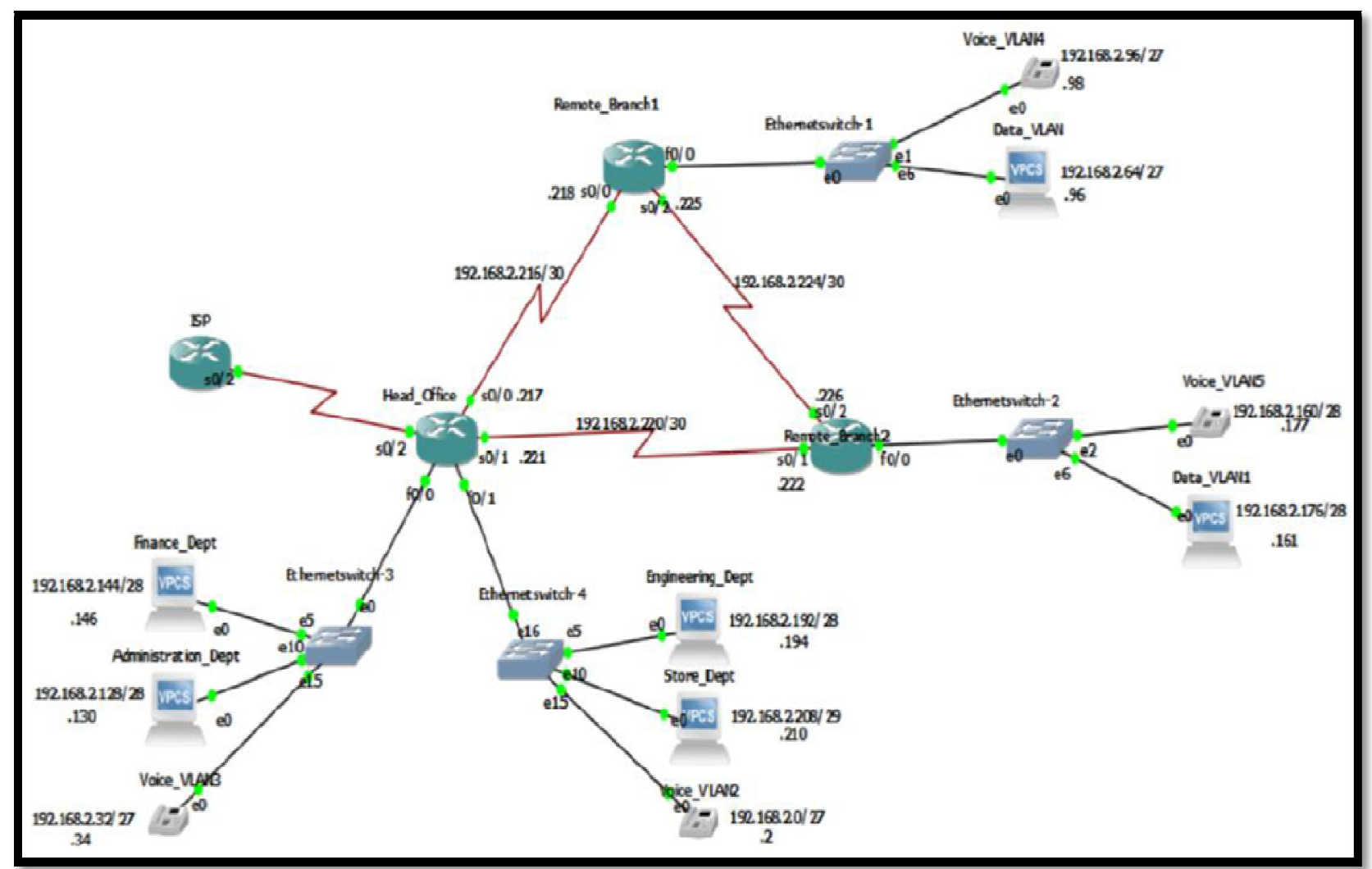

Figure 1: Wide Area Network Design

The GNS3 software was used design of the Wide Area Network (WAN) for this study as shown above in Fig 1. Each network domain as part of the WAN was segmented into smaller networks as Virtual Local Area Network (VLANs). The purpose of the VLANs is to reduce congestion and improves security. The routing protocols RIP, EIGRP and OSPF are configured on the WAN (the network domain node: Remote_Branch1, Head_Office and Remote_Branch2 routers). After each configuration the nodes build a routing table which it uses to determine routes. The Figure 2 shows a route trace from the Finance Dept (192.168.2.146/28) to the Data VLAN1 $(192.168 .2 .161 / 28)$ work stations. The traffic is routed via the Remote Branch2 (interface s0/ 1-192.168.2.221 and s0/ 2-192.168.2.222) to the destination VLAN via the Head Office router. When the interface $\mathrm{s} 0 / 1$ or $\mathrm{s} 0 / 2$ is shut down the convergence is broken and a re-convergence is established via the Remote_Branch2 as shown in Figure 3.

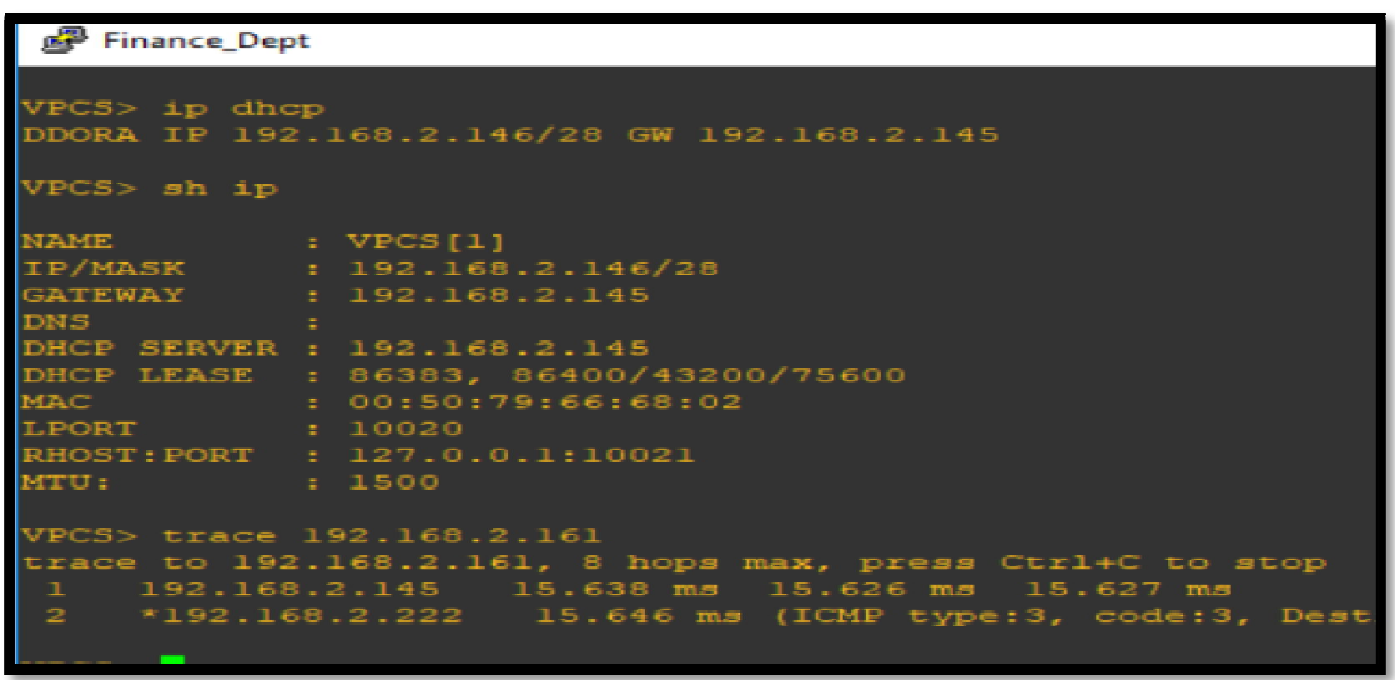

Figure 2: Trace Route from Finance Dept to the Data_VLAN1 via Head Office and Remote_Branch1 Routers 


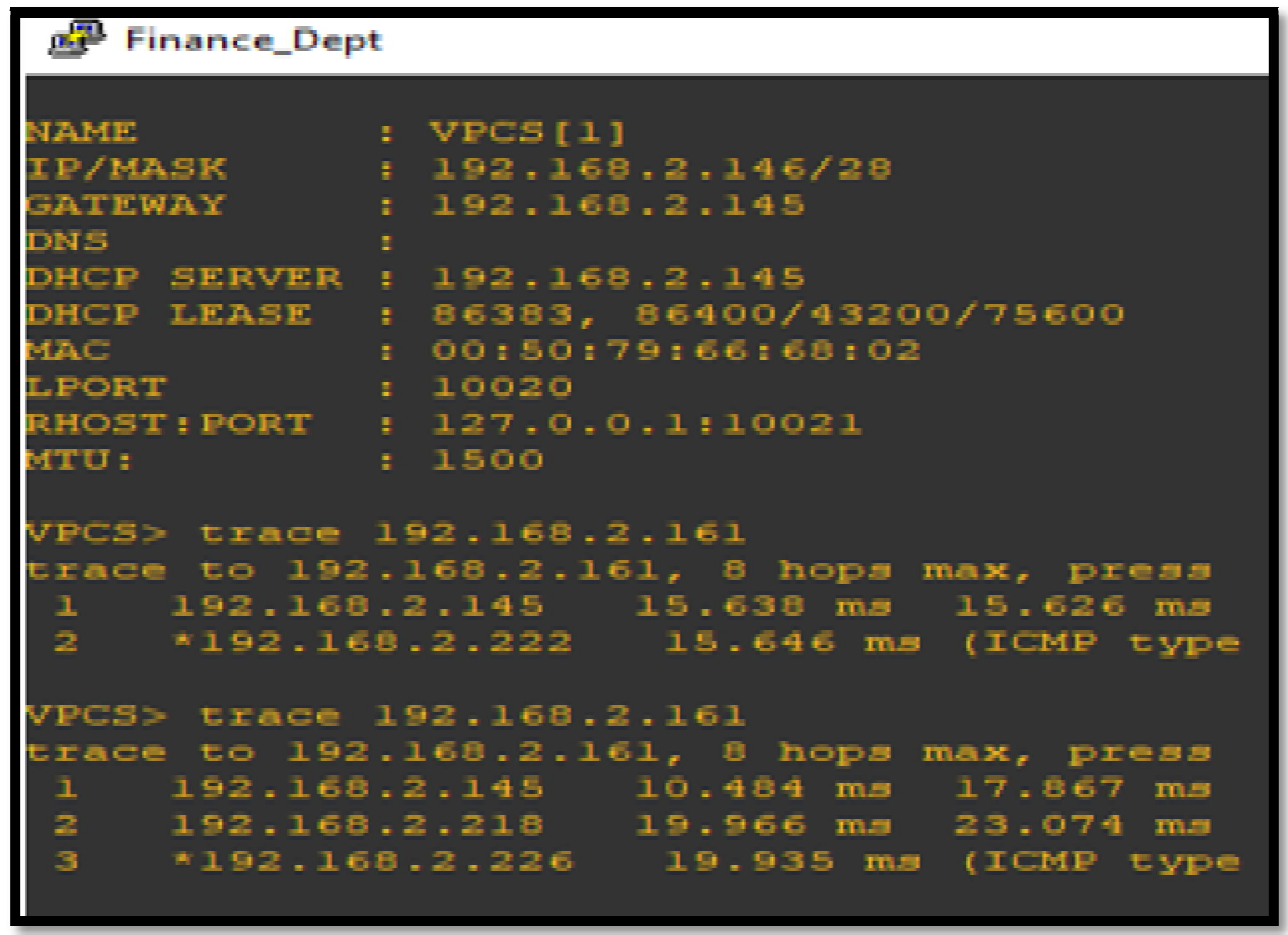

Figure 3: Trace Route from Finance Dept to the Data_VLAN1 via Head Office and Remote_Branch2 Routers

During the elapsed time for the re-convergence to be established packets are loss. Thus, communication via from the source to the destination would halted till the new convergence is formed as per the routing protocol configured be it EIGRP, OSPF or RIP as shown in Figure 4.

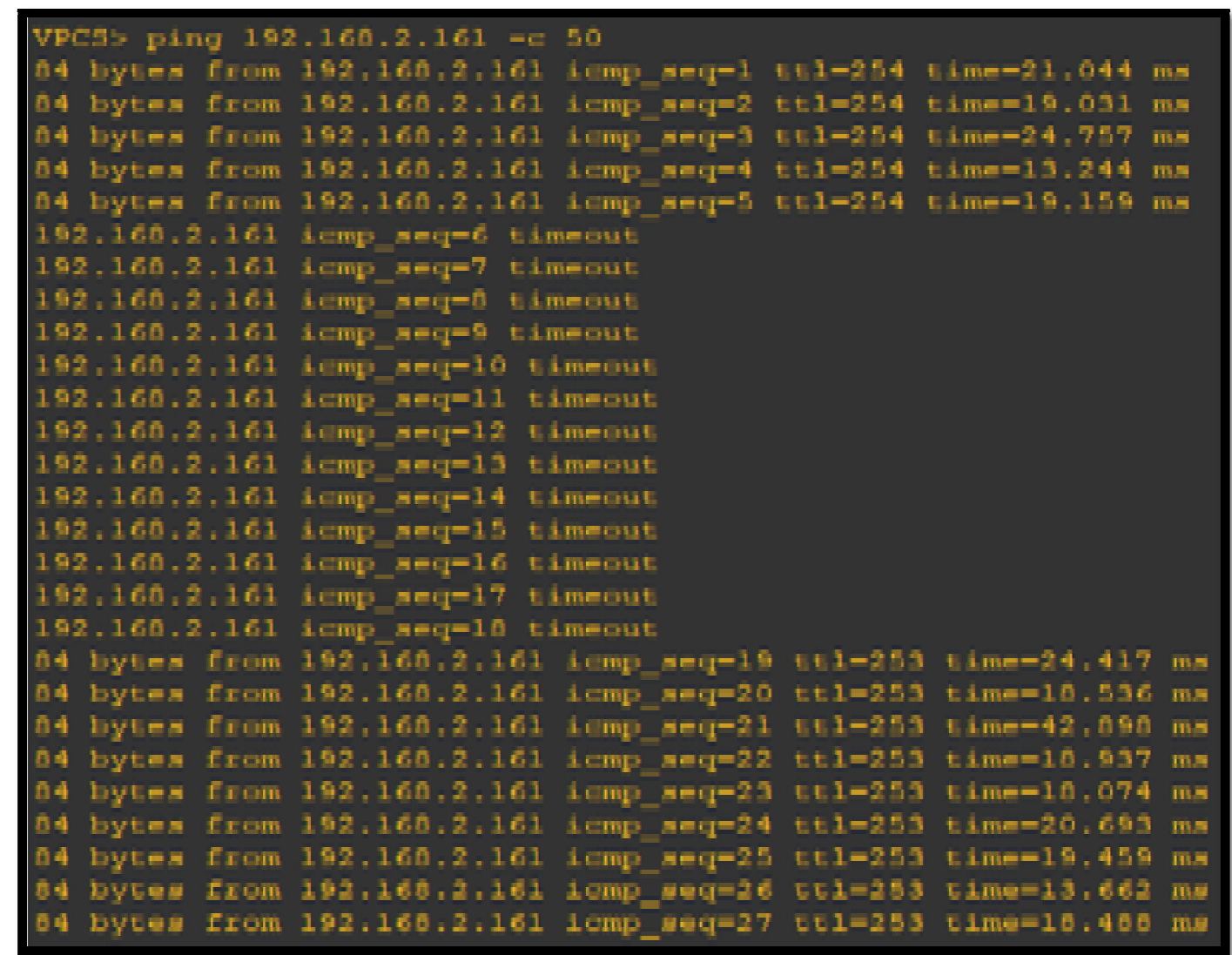

Figure 4: Packet Loss after Link Shutdown 


\section{Results and Discussion}

The test case for this study is to repeat the trace route and edge (link) shutdown processes for the routing protocols EIGRP, OSPF and RIP in order to find which one has the quickest re-convergence time with minimal packet loss. The Table 1 summarizes the test results for EIGRP routing protocol. At the end of the 5 different tests carried out under the EIGRP Protocol, the average test results revealed that 12 packets of data were lost after shutdown and the time taking to re-converge was $5.294 \mathrm{~ms}$.

\begin{tabular}{|c|c|c|}
\hline Test & $\begin{array}{c}\text { No. of Packets Lost } \\
\text { After Shutdown }\end{array}$ & $\begin{array}{c}\text { Time Taken to } \\
\text { Re-Converge (ms) }\end{array}$ \\
\hline 1 & 13 & 5.258 \\
\hline 2 & 12 & 6.106 \\
\hline 3 & 14 & 4.834 \\
\hline 4 & 10 & 7.228 \\
\hline 5 & 10 & 3.044 \\
\hline Average & 12 & 5.294 \\
\hline
\end{tabular}

Table 1: Results of the 5 Test Carried Out on EIGRP

The Table 2 summarizes the test results for OSPF routing protocol. At the end of the 5 different tests carried out under the OSPF Protocol, the average test results revealed that 4 packets of data were lost after shutdown and the time taking to reconverge was $2.0408 \mathrm{~ms}$.

\begin{tabular}{|c|c|c|}
\hline Test & $\begin{array}{c}\text { No. of Packets Lost } \\
\text { After Shutdown }\end{array}$ & $\begin{array}{c}\text { Time Taken to } \\
\text { Re-converge (ms) }\end{array}$ \\
\hline 1 & 3 & 1.965 \\
\hline 2 & 4 & 1.216 \\
\hline 3 & 4 & 2.529 \\
\hline 4 & 3 & 4.019 \\
\hline 5 & 4 & 0.484 \\
\hline Average & 4 & 2.0408 \\
\hline
\end{tabular}

Table 2: Results of the 5 Test Carried Out on OSPF

The Table 3 summarizes the test results for RIP routing protocol. At the end of the 5 different tests carried out under the RIP Protocol, the average test results revealed that 30 packets of data were lost after shutdown and the time taking to reconverge was $10.760 \mathrm{~ms}$.

\begin{tabular}{|c|c|c|}
\hline Test & $\begin{array}{c}\text { No. of Packets Lost } \\
\text { After Shutdown }\end{array}$ & $\begin{array}{c}\text { Time Taken to } \\
\text { Re-converge (ms) }\end{array}$ \\
\hline 1 & 26 & 9.524 \\
\hline 2 & 30 & 10.984 \\
\hline 3 & 31 & 11.264 \\
\hline 4 & 35 & 12.132 \\
\hline 5 & 29 & 9.895 \\
\hline Average & 30 & 10.760 \\
\hline \multicolumn{2}{|c|}{ Table 3: Results of the 5 Test Carried Out on OSPF } \\
\hline
\end{tabular}

In summary from the Figure below it can be deduced that the routing protocol with the least number of packets of data lost during shutdown is the Open Shortest Path First (OSPF) routing protocol and it has the lowest time to re-converge. Also, Enhanced Interior Gateway Routing Protocol (EIGRP) follows with the second least number of data packets lost after shutdown and takes $5.294 \mathrm{~ms}$ to converge which is higher than that of OSPF. Lastly, the routing protocol with the highest number of packets of data lost after shutdown is the Routing Information Protocol with $10.763 \mathrm{~ms}$. 


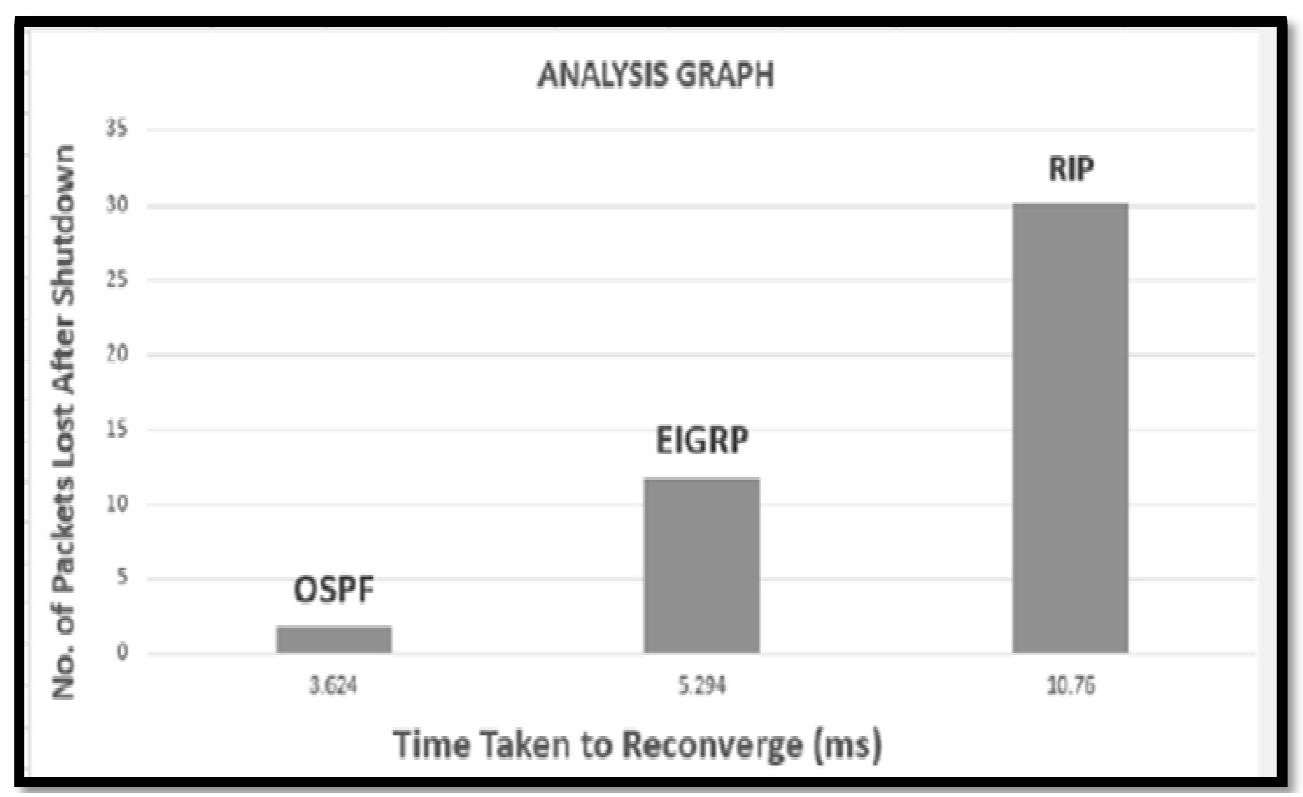

Figure 5: OSPF, EIRGP and RIP Re-Convergence Ability Analysis Graph

\section{Conclusions and Recommendation}

This study set out to determine the re-convergence ability of the routing protocols EIGRP, OSPF and RIP with minimum packet loss and elapsed time. The results of the study shows that, the OSPF routing protocol has the optimal reconvergence elapsed time with minimal packet loses followed by IEGRP and RIP. Taking together these results it could be suggested that in critical WAN where continuous connectivity is a must the ideal routing protocols for such consideration should be OSPF compared to EIGRP and RIP. The study has gone some way towards enhancing our understanding of routing protocols capability for WAN routing applications. The current study has only considered RIP, OSPF and EIGRP. More research is required to determine the efficacy of other than the considered in this study in terms re-convergence metric.

\section{References}

i. Bansal, A., \& Goel, P. (2017). Configuration and detailed analysis of Enhanced Interior Gateway Routing Protocol (EIGRP) using Cisco Packet Tracer. International Journal of Advanced Research, 5(7), 422-431.

ii. Cho, T., \& Lee, J. (2011). The study on the OLSRP (Optimized Link State Routing Protocol) implementation in the mobile ad-hoc network. The Transactions of the Korean Institute of Electrical Engineers, 60(4), 257-261.

iii. Gouveira, L., Pioro, M., \& Rak, J. (2016), Static and dynamic optimization models for network routing problems. Network, 69(1), 3-5.

iv. Hussain, M.W., \& Jamwal, S. (2016), Comparative analysis of various routing protocols. International Journal of Modern Engineering Research. 6(3), 67-73.

v. Lee, B., Cai, W., Turner, S., \& Koh, J. (2001). Comparison of network protocol and architecture for distributed virtual simulation environment. ACM SIGOPS Operating Systems Review, 35(3), 30-42.

vi. Quoitin, B., \& Uhlig, S. (2005). Modeling the routing of an autonomous system with C-BGP. IEEE Network journal, 19(6), 12-19.

vii. Rakheja, P., Kaur, P., Gupta, A., \& Sharma, A. (2012). Performance analysis of RIP, OSPF, IGRP and EIGRP routing protocols in a network. International Journal of Computer Applications, 48(18), 6-11.

viii. Rathi, B., \& Singh, E. F. (2015). Performance analysis of distance vector and link state routing protocols. International Journal of Computer Science Trends and Technology, 3(4), 23-32.

ix. Tewari, A., \& Kumar, A. (2014). Different routing algorithm for computer networks. ACM SIGMETRICS Performance Evaluation Review Journal, 1(1), 120-125.

x. Verma, A., \& Bhardwaj, N. (2016). A review on Routing Information Protocol (RIP) and Open Shortest Path First (OSPF) routing protocol. International Journal of Future Generation Communication and Networking, 9(4), 161-170.

xi. Wang, J., Lui, J., \& Wang, X, (2011). Performance analysis of multicast routing algorithm based on network coding. Journal of Electronics and Information Technology, 30(11), 2605-2608. 